\title{
Kimura Disease: An Unusual Presentation in Paediatric Age Group
}

\author{
ZA Ibrahim*, MPath, KL Pan**, FRCS, SL Wong***, MMed (Rad), PS Shanmugam**, MD, \\ AN Zulkarnaen*, MPath \\ *Department of Pathology, Universiti Malaysia Sarawak, Kuching, Malaysia \\ **Department of Orthopaedics, Universiti Malaysia Sarawak, Kuching, Malaysia \\ ${ }^{* * *}$ Department of Radiology, Universiti Malaysia Sarawak, Kuching, Malaysia
}

\begin{abstract}
Kimura disease is a chronic inflammatory disorder that is prevalent among Asians. It rarely affects the upper limbs or paediatric patients. We report a case of Kimura disease with first presentation as a soft tissue mass in the arm mimicking a soft tissue neoplasm with metastasis in a 12 year old Chinese boy.
\end{abstract}

Key Words:

Kimura Disease, Soft Tissue Tumour, Arm

\section{INTRODUCTION}

Kimura disease (KD) commonly presents with stretching dermis and a subcutaneous painless nodular mass and typically affects the head and neck region. A common area of initial presentation lies in the infra-auricular and retroauricular area and is often accompanied by increased major salivary glands mass and regional lymphadenopathy. Upper limb involvement is rare in $\mathrm{KD}$ particularly among paediatric patients. This disease is most commonly seen among young adults aged 27 to 40 years old. The aim of this report is highlight the clinical presentation, diagnosis and brief overview of treatment involving this unusual $\mathrm{KD}$ presentation affecting the upper limb of a paediatric patient.

\section{CASE REPORT}

A 12-year-old boy presented with a slowly enlarging mass on his right arm that had been initially noted at one year of age. There was no history of known previous medical illness and the boy was otherwise well. The mass was non-tender,had a smooth outer surface, was located at the medial aspect of the arm (Figure 1), and measured $4 \times 3 \times 3 \mathrm{~cm}$. Plain $X$ ray showed a subcutaneous oval shaped soft tissue lesion with no visible calcification within the lesion. The ipsilateral humerus bone was unremarkable with no evidence of erosion or destruction. This was not likely to be a vascular lesion (e.g. arteriovenous malformation or haemangioma) as there were no phleboliths noted.
The impression was a non-specific soft tissue lesion that warranted further assessment by magnetic resonance imaging (MRI). MRI showed a fusiform shaped soft tissue subcutaneous mass in the medial compartment of the arm displacing the arm muscles laterally.The lesion was hyperintense on $\mathrm{T} 2$ weighted image (WI) and exhibited higher signal intensity than muscle on T1WI with avid gadolinium enhancement. There were flow void channels noted coursing through the long axis of the lesion (Figure 2). Subcutaneous oedema was noted in an adjacent area.

An enlarged right axillary lymph node was also detected clinically and on MRI. Clinically the lymph node was nontender, firm and mobile.Biopsy of the arm mass and fine needle aspiration of the axillary lymph node showed features of KD (Figure 3 ). The arm mass was eventually surgically resected (Figure 4). No other treatment was administered. Six months after the surgery, the arm wound had healed well with (no residual mass) and only some residual surrounding indurations. The axillary mass remained palpable with no significant change in size. The boy was otherwise well and remained under close follow-up. No signs or symptoms of nephrotic syndrome were seen and urinalysis was unremarkable.

\section{DISCUSSION}

$\mathrm{KD}$ is endemic in Asia and it may occur sporadically in other regions ${ }^{1}$. This disease commonly affects young adults (mean age at presentation is $28 \mathrm{y})^{1}$. In a study on Kimura's disease in children by Vishwantha, all patients presented with head and neck swelling without upper limb involvement ${ }^{1}$. Fifty per cent of these patients had post auricular involvement ${ }^{1}$.

Kimura disease in the upper limb presents as a slow growing lesion and may remain unchanged for years ${ }^{2}$. MRI findings for the patient discussed in this report were consistent with Kimura disease including a subcutaneous fat lesion with higher signal intensity compared to muscle on both $\mathrm{T} 1$ and T2 weighted images. Pathognomonic MRI findings included flow void area corresponding to vascular channels within the 


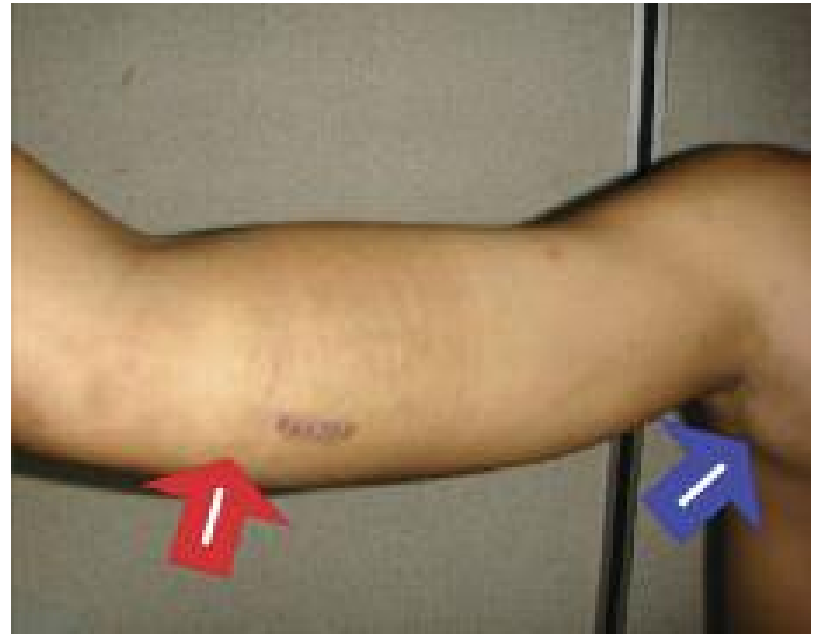

Fig. 1: The arm swelling and the axillary lymphadenopathy There was a fusiform swelling noted at the medial aspect of the right arm. The mass displayed smooth outer surface (red arrow). An enlarged rounded swelling at the axillary fossa was shown by the blue arrow. A biopsy scar was also seen.

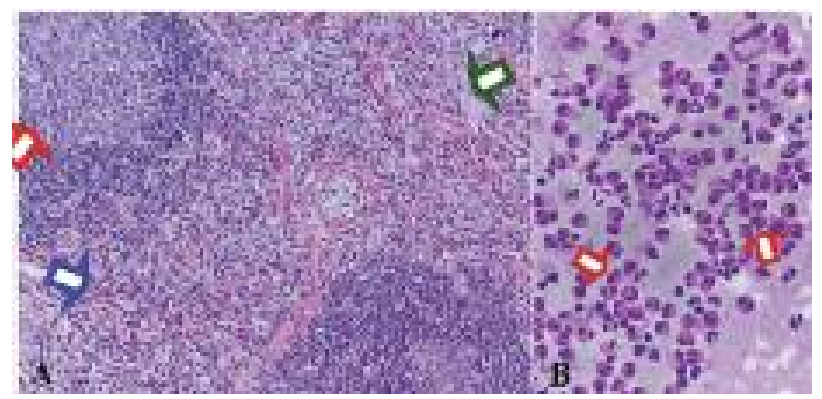

Fig. 3: Microscopic (A) and cytological (B) features of the arm mass (H\&E 20X)

A: There are two hyperplastic lymphoid follicles with prominent germination centers (red arrow). Numerous peri-follicular clusters of eosinophils (blue arrow) and small arteries hyperplasia (green arrow). No granuloma or multinucleated giant cell detected. B: There are numerous eosinophils (red arrows) in a background of scattered lymphoid cells of various sizes (reactive hyperplasia features).

lesion with surrounding soft tissue oedema ${ }^{3}$. KD may also present with eosinophilia, elevated erythrocyte sedimentation rate (ESR) and increased immunoglobulin E (IgE) and such laboratory analyses may be helpful as part of the diagnostic process ${ }^{2}$. However, these features were not seen in our case.

Although the pathogenesis of KD is poorly understood, the disease is known to be associated with allergy, trauma, autoimmune disease and some systemic illnesses ${ }^{4}$. Currently, $\mathrm{KD}$ is regarded as an allergic disease, systemic immunological disorder ${ }^{4}$. T helper-2 cell proliferation and overexpression of cytokines may play an important role in KD pathogenesis ${ }^{4}$.
A
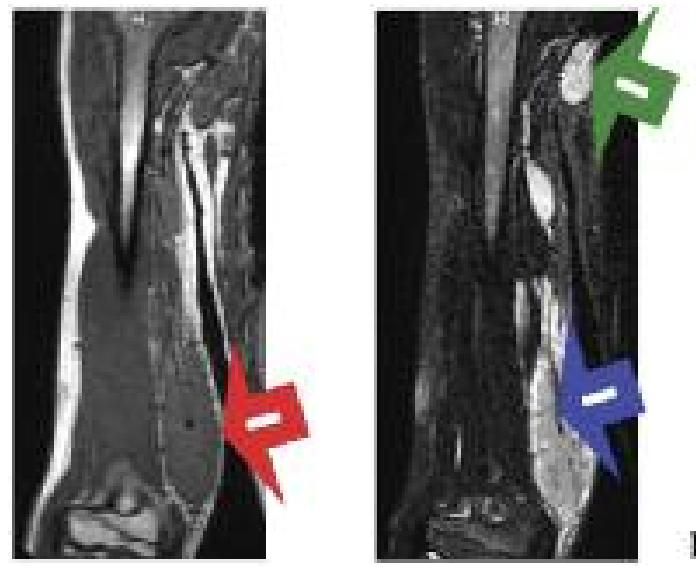

Fig. 2: $M R I$ image of the arm lesion Coronal T1WI (A): Red arrow indicated the fusiform lesion with hyperintense signal than the muscle. Coronal fat suppressed T2WI (B): The blue arrow showing the flow void channels within the lesion. The green arrow highlighted the enlarged axillary lymph node.

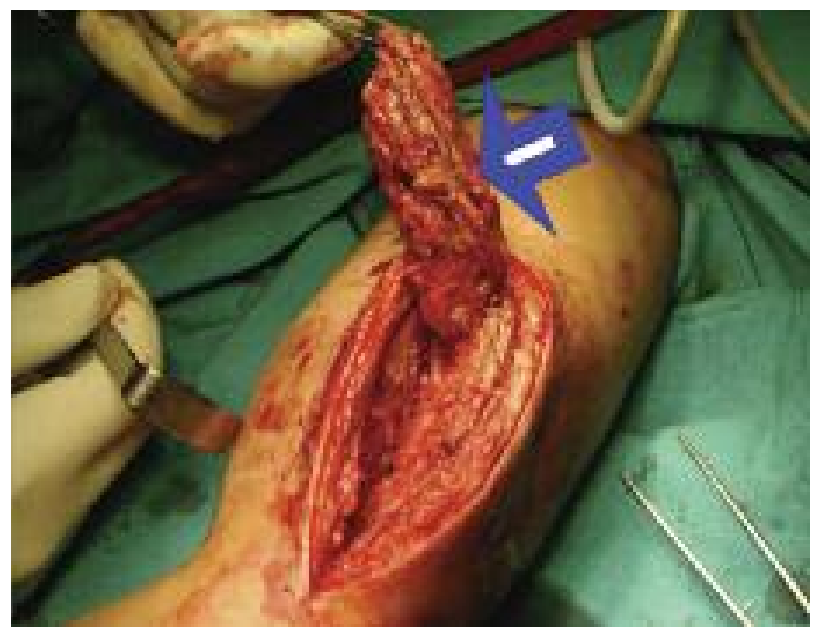

Fig. 4: The surgically resected arm mass

The mass was located at deep subcutaneous layer pressing down onto the underlying muscle. No adjacent muscle involvement found. The mass was firm and rubbery with irregular ill-defined outer surface. Morphologically, the mass resemble a soft tissue malignancy.

Angiolymphoid hyperplasia with eosinophilia (ALHE) is a well-known entity, which histologically and clinically mimics KD. However $\mathrm{KD}$ is differentiated by regional lymph node involvement, the presence of lymphoid tissue with prominent germination centre and the absence of endothelial atypia ${ }^{4} \mathrm{KD}$ is a predominantly lymphoid follicle disorder whilst ALHE is a vascular disorder ${ }^{4}$.

The presence of abundant eosinophils in fine needle aspiration cytology (FNAC) of a lesion may lead to other differential diagnoses including Hodgkin disease and allergic reaction due to insect bites or vaccination ${ }^{4}$. Definitive diagnosis is ascertained by tissue biopsy, but FNAC is helpful in detecting $\mathrm{KD}$ recurrence. 
Treatments for KD are frequently unsatisfactory. New lesions may occur and recurrence after resection is common ${ }^{5}$. Many therapeutic modalities that have been used to treat KD are associated with relapse ${ }^{2}$. A definitive $\mathrm{KD}$ treatment policy has never been determined, although Chang et al reported that radiotherapy is more effective against Kimura's disease in comparison to local surgical excision or administration of systemic steroids ${ }^{3}$. Recurrence rate occurs more with steroid therapy and some claim that surgery is the best modality of treatment ${ }^{1,4}$. Other treatments include administration of imatinib, cyclosporine, isotretinoin and cryotherapy ${ }^{4}$. Kidney involvement has been observed; in fact, nephrotic syndrome is a common systemic complication in paediatric KD cases ${ }^{1}$. We opted for surgical treatment in the present case in view of the indolent properties of the lesion and the age of the patient.
$\mathrm{KD}$, albeit rare, should be considered in cases with subcutaneous nodules that are associated with regional lymph node enlargement, especially in young Asians including those of paediatric age. Distinctive MRI findings certainly help to narrow the differential diagnoses. A highindex of suspicion accompanied by tissue biopsy confirmation is essential to diagnose this inflammatory condition, which is known to mimic neoplasm.

\section{ACKNOWLEDGEMENT}

The authors wish to thank Dr.Dayangku Norlida A Ojep and Angela Chia Yin Yin for their assistance in preparing this report. 


\section{REFERENCES}

1. Viswanatha B. Kimura's disease in children: A 9 years prospective study. Int J Pediatr Otorhinolaryngol 2007; 71(10): 1521-5.

2. Sun QF, Xu DZ, Pan SH, Ding JG, Xue ZQ, Miao CS et al. Kimura disease: review of the literature. Intern Med J 2008; 38(8): 668-72.

3. Chang AR, Kim K, Kim HJ, Kim IH, Park CI, Jun YK. Int J Radiat Oncol Biol Phys 2006; 65(4): 1233-9.

4. Briggs PL. Kimura disease is not angiolymphoid hyperplasia with eosinophilia: Clinical and pathological correlation with literature review and definition of diagnostic criteria. An Bras Dermatol 2006;(2): 167-73.

5. Fletcher CDM. (2007). Diagnostic histopathology of tumours. 3rd Ed. Churchill Livingstone Elsevier. 